\title{
EL FORO DE CONSUMIDORES: COMENTARIOS A LA SENTENCIA DEL TJUE DE 23 DE DICIEMBRE DE 2015, RÜDIGER HOBOHM C. BENEDIKT KAMPIK LTD \& CO. KG Y OTROS, C-297/14
}

\author{
JURISDICTION IN RESPECT OF CONSUMER CONTRACTS: \\ COMMENTS TO THE CJEU JUDGEMENT OF 23RD DECEMBER \\ 2015, RÜDIGER HOBOHM V. BENEDIKT KAMPIK LTD \& CO. \\ KG AND OTHERS, C-297/14
}

\author{
Ma José Castellanos Ruiz \\ Profesora Visitante de Derecho Internacional Privado \\ Universidad Carlos III de Madrid
}

Recibido: 15.07.2017 / Aceptado: 15.09.2017

DOI: https://doi.org/10.20318/cdt.2017.3893

\begin{abstract}
Resumen: La Sentencia del TJUE 23 diciembre 2015, Rüdiger Hobohm c. Benedikt Kampik Ltd \& Co. $K G$ y otros, C-297/14, recoge la interpretación del art. 15.1.c) del Reglamento 44/2001, en relación con el art. 16.1 de dicho instrumento, preceptos que contienen el foro de consumidores. Aunque el TJUE ha interpretado estos preceptos en variada jurisprudencia, la petición de decisión prejudicial se realiza para determinar si este foro puede operar en relación con un segundo contrato, cuando se trata de dos contratos referidos a distintos servicios y separados en el tiempo, pero con una cierta conexión, y de los cuales sólo el primero se ha celebrado directamente en el ámbito de las actividades que el profesional dirige hacia el Estado miembro del domicilio del consumidor.
\end{abstract}

Palabras clave: Reglamento 44/2001, foro de consumidores, concepto de "actividad comercial o profesional dirigida", concepto de "nexo estrecho".

Abstract: The CJEU Judgement of 23rd December 2015, Rüdiger Hobohm c. Benedikt Kampik Ltd \& Co. KG y otros, C-297/1, refers to the interpretation of art. 15.1.c) of Regulation 44/2001, read in conjunction with article 16.1 of that regulation, precepts that contain the jurisdiction in respect of consumer contracts. Althoug the CJEU has interpreted this precepts on several occasions, the request for a preliminary ruling involves a situation in which there are two contracts of different dates relating to different services, but they are, to a certain extent, connected. The Court must decide whether a consumer can bring an action against a professional in the courts of the consumer's domicile in relation to a second contract which does not come directly within the scope of the activity which the contractual partner directs to the consumer's Member State of residence.

Keywords: Regulation 44/2001, jurisdiction in respect of consumer contracts, concept of "commercial or professional activity directed", concept of "close link".

Sumario: : I. Introducción de la STJUE 23 diciembre 2015, Rüdiger Hobohm c. Benedikt Kampik Ltd \& Co. KG y otros, C-297/14: 1. Hechos. 2. Cuestión prejudicial. II. Análisis de la STJUE 23 diciembre 2015, Rüdiger Hobohm c. Benedikt Kampik Ltd \& Co. KG y otros, C-297/14: 1. Los requisitos para la aplicación del artículo 15.1.c) del Reglamento 44/2001 (art. 17.1.c) del Reglamento 1215/2012). 2. El concepto de "actividad dirigida". 3. Los elementos constitutivos de "nexo estrecho". III. Conclusiones. 


\section{Introducción de la STJUE 23 diciembre 2015, Rüdiger Hobohm c. Benedikt Kampik Ltd \& Co. $K G$ y otros, C-297/14}

1. La STJUE 23 diciembre 2015, Rüdiger Hobohm c. Benedikt Kampik Ltd \& Co. KG y otros, C-297/14, fue dictada como respuesta a la cuestión prejudicial planteada por el Bundesgerichtshof -Tribunal Supremo Civil y Penal, Alemania-, en el marco de un litigio entre el Sr. Hobohm, con domicilio en Alemania, y Benedikt Kampik Ltd \& Co. KG, el Sr. Kampik y Mar Mediterraneo Werbe- und Vertriebsgesellschaft für Immobilien, S.L., con domicilio en España, en relación con la devolución de los importes entregados al Sr. Kampik por el Sr. Hobohm.

La cuestión prejudicial tenía por objeto la interpretación del artículo 15, apartado 1, letra c), del Reglamento (CE) núm. 44/2001 del Consejo, de 22 de diciembre de 2000 (DO núm. L 12, 16 enero 2001), relativo a la competencia judicial, el reconocimiento y la ejecución de resoluciones judiciales en materia civil y mercantil, en relación con el artículo 16, apartado 1, de dicho Reglamento; que será denominado en adelante Reglamento 44/2001².

\section{Hechos}

2. Durante el año 2005 el Sr. Kampik, que desarrollaba su actividad profesional en España, intermedió entre el Sr. Hobohm y Kampik Immobilien KG para la adquisición de un apartamento en un complejo de vacaciones situado en Denia (Alicante), el cual sería construido por un promotor alemán. Se debe resaltar que dichos apartamentos de vacaciones se comercializaban, entre otros países, en Alemania, a través de un folleto publicitario redactado en alemán.

Como consecuencia del contrato de intermediación celebrado entre las partes arriba señaladas, el 17 de junio de 2006, el promotor del complejo de vacaciones, en calidad de vendedor, y el Sr. Hobohm y su esposa, en calidad de compradores, firmaron un contrato de compraventa del apartamento situado en Denia. Aunque el matrimonio Hobohm realizó el pago de los dos primeros plazos del precio de compra del apartamento por importe de 62490 euros, en el año 2008 el promotor atravesó dificultades financieras que obstaculizaban la terminación de la construcción del complejo de vacaciones.

3. Como consecuencia de los hechos relacionados, el Sr. Kampik ofreció entonces al Sr. Hobohm encargarse de conseguir la habitabilidad de la vivienda. Por esta razón el matrimonio Hobohm se desplazó a España y otorgó a favor del Sr. Kampik un poder notarial para la defensa de sus intereses en relación con el contrato de compraventa, es decir, que las partes celebraron un contrato de mandato.

El Sr. Hobohm extendió al Sr. Kampik un cheque al portador por importe de 27647 euros, en concepto de parte del tercer plazo del precio de compra del apartamento, que el Sr. Kampik ingresó en la cuenta de Mar Mediterraneo Werbe- und Vertriebsgesellschaft für Immobilien, S.L. Pero además, en el año 2009, el Sr. Hobohm transfirió al Sr. Kampik un importe adicional de 1448,72 euros.

4. Tras una serie de desavenencias entre las partes del contrato de mandato relativas a la insolvencia del promotor, el matrimonio Hobohm revocó el poder otorgado a favor del Sr. Kampik. Y posteriormente, el Sr. Hobohm presentó demanda ante el Landgericht Stade -Tribunal Regional Civil y Penal de Stade, Alemania-, en cuya jurisdicción se encontraba su domicilio, para obtener la devolución de los importes realizados al Sr. Kampik.

Sin embargo, dicho órgano jurisdiccional declaró la inadmisibilidad de la demanda, mediante resolución de 21 de septiembre de 2011, por considerar que carecía de competencia territorial.

\footnotetext{
1 STJUE 23 diciembre 2015, Rüdiger Hobohm c. Benedikt Kampik Ltd \& Co. KG yotros, C-297/14 (ECLI:EU:C:2015:844).

2 Desde el 10 de enero de 2015, el Reglamento 44/2001 ha sido sustituido por el Reglamento (UE) núm. 1215/2012 del Parlamento Europeo y del Consejo, de 12 de diciembre de 2012 (DO núm. L 351, 20 diciembre 2012), relativo al reconocimiento y la ejecución de resoluciones judiciales en materia civi y mercantil. En las referencias posteriores que se hagan en relación con dicho instrumento internacional, éste será denominado Reglamento 1215/2012.
} 
5. El Sr. Hobohm presentó recurso de apelación ante el Oberlandesgericht Celle -Tribunal Superior Regional Civil y Penal de Celle- contra la resolución antes mencionada, pero fue desestimado el 18 de julio de 2012. Dicho órgano jurisdiccional de apelación consideró inaplicable el artículo 15.1.c), del Reglamento 44/2001 y, por consiguiente, la competencia del foro del domicilio del $\mathrm{Sr}$. Hobohm, por estimar que el contrato de mandato no podía relacionarse directamente con la actividad de intermediación inmobiliaria "dirigida" por el Sr. Kampik "a" Alemania, en el sentido de dicha disposición.

6. A continuación, el Sr. Hobohm decidió recurrir en casación ante el Bundesgerichtshof -Tribunal Supremo Civil y Penal- contra la resolución dictada en apelación. Dicho órgano jurisdiccional consideró que en concreto la actividad de intermediación inmobiliaria ejercida por el Sr. Kampik en España estaba "dirigida a" Alemania, en el sentido de dicha disposición, puesto que concurrían algunos de los indicios de las actividades "dirigidas al" Estado miembro del domicilio del consumidor que se detallan en la sentencia Pammer y Hotel Alpenhof: a) el Sr. Kampik ofrecía sus servicios en Internet a través de un sitio registrado con un nombre de dominio de primer nivel ".com", que estaba redactado en alemán; b) se indicaba en este sitio una dirección electrónica de contacto alojada en un servidor que utilizaba un nombre de dominio de primer nivel ".de"; c) para contactar con el servicio de gestión de la actividad profesional del Sr. Kampik debía utilizarse un número de teléfono de Berlín; y d) el Sr. Kampik empleaba para su actividad folletos redactados en alemán ${ }^{3}$. El contrato de intermediación cumplía, pues con los requisitos para la aplicación del artículo 15.1.c), del Reglamento 44/2001, al haberse celebrado en el ejercicio de la actividad del Sr. Kampik "dirigida al" Estado miembro del domicilio del Sr. Hobohm. Sin embargo, según el Bundesgerichtshof -Tribunal Supremo Civil y Penal- , si se analiza de forma separada el contrato de mandato en el que se basaban las pretensiones del Sr. Hobohm, éste no cumplía los requisitos para la aplicación de dicha disposición, puesto que no se enmarcaba dentro de tal actividad.

Por otro lado, el mencionado órgano jurisdiccional señaló que existía un nexo material decisivo entre la actividad de intermediación inmobiliaria "dirigida" por el Sr. Kampik "a" Alemania y la celebración del contrato de mandato. Fue precisamente esta actividad la que llevó a a la celebración por el matrimonio Hobohm, en los años 2005 y 2006, del contrato de intermediación y del contrato de compraventa. Pero si no existieran estos contratos, no se hubiera celebrado el contrato de mandato que fundamenta las pretensiones del Sr. Hobohm y que tenía como objetivo la resolución de los problemas de ejecución del contrato de compraventa. Según el Bundesgerichtshof -Tribunal Supremo Civil y Penal-, aun cuando las obligaciones de las partes en virtud del contrato de intermediación se cumplieron mediante la celebración del contrato de compraventa, no se consiguió el objetivo económico del contrato de intermediación, que no era otro que el matrimonio Hobohm tuviera el disfrute efectivo del apartamento conseguido gracias a la actividad de intermediación.

\section{Cuestión prejudicial}

7. Como consecuencia de los hechos relacionados, el Bundesgerichtshof -Tribunal Supremo Civil y Penal- decidió suspender el procedimiento y plantear al Tribunal de Justicia la siguiente cuestión prejudicial:

“¿Puede un consumidor, en virtud del artículo 15, apartado 1, letra c), segunda alternativa, en relación con el artículo 16, apartado 1, segunda alternativa, del Reglamento $n^{\circ} 44 / 2001$ [...], interponer una demanda ante el tribunal del lugar de su domicilio contra la otra parte contratante, quien ejerce actividades comerciales o profesionales en otro Estado miembro de la Unión Europea, cuando, si bien el contrato en el que se fundamenta la demanda no está directamente comprendido en el ámbito de la actividad del socio contractual dirigida al Estado miembro del domicilio del consumidor, dicho contrato tiene

3 STJUE 7 diciembre 2010, Peter Pammer c. Reederei Karl Schlüter GmbH \& Co. KG y Hotel Alpenhof GesmbH c. Oliver Heller, C-585/08 y C-144/09, Rec. 2010, p. I-12527 (ECLI:EU:C:2010:740), apartados 83, 84, 93 y 94. 
por objeto conseguir el éxito económico perseguido con otro contrato celebrado anteriormente entre las partes y ya ejecutado, que sí está comprendido en el ámbito de aplicación de las disposiciones citadas?"”.

\section{Análisis de la STJUE 23 diciembre 2015, Rüdiger Hobohm c. Benedikt Kampik Ltd \& Co. KG y otros, C-297/14}

8. La petición de decisión prejudicial del Bundesgerichtshof plantea la posibilidad de acudir al foro de consumidores del Reglamento 44/2001 en una situación en la que, tras haberse concluido y ejecutado un contrato de intermediación para la adquisición de un inmueble en España - actividad que el profesional dirigía a Alemania a través de su publicidad - , se ha concluido un contrato de mandato para solucionar algunos problemas relacionados con el contrato de compraventa de dicho inmueble. En cuyo caso, tendrían que declararse competentes los tribunales alemanes, en virtud del art. 16.1 del Reglamento $44 / 2001$, pues el domicilio del consumidor se encuentra en Alemania, ya que al ser considerada la parte débil en el contrato, éste tiene la posibilidad de litigar ante los tribunales de su domicilio, los tribunales alemanes, $o$ ante los tribunales del domicilio de la otra parte contratante, los tribunales españoles ${ }^{5}$.

En principio, es preciso que para poder acudir al foro de consumidores en virtud del art. 16.1 del Reglamento 44/2001, el contrato de mandato tiene que cumplir con los requisitos establecidos en el art. 15.1.c) del Reglamento 44/20016.

9. E1 Tribunal de Justicia ya ha interpretado los arts. 15.1.c) y 16.1 del Reglamento 44/2001 en varias ocasiones, concretamente en las sentencias Pammer y Hotel Alpenhof, Mühlleitner, Emrek y Maletic ${ }^{7}$.

La diferencia entre los asuntos citados y el que nos ocupa, es que mientras que en las sentencias citadas se trataba de un único contrato, el asunto objeto de análisis presenta la particularidad de que se trata de una situación en la que existen varios contratos referidos a distintos servicios y separados en el tiempo, pero con una cierta conexión. Concretamente, la jurisdicción nacional pregunta al Tribunal de Justicia sobre la posibilidad de que un consumidor interponga ante el tribunal del lugar de su domicilio una demanda contra un profesional en relación con un contrato que no está directamente comprendido en el marco de la actividad que el profesional dirige al Estado miembro de residencia del consumidor, pero que tiene como finalidad conseguir el éxito económico de un contrato celebrado con anterioridad por las partes, cuando este contrato sí se enmarca dentro del ámbito de dicha actividad.

Tal y como se recoge en las Conclusiones del Abogado General, Pedro Cruz Villalón, el problema se centra pues en la cuestión de si los requisitos enunciados por el artículo 15.1.c) del Reglamento 44/2001, pueden considerarse cumplidos a través de la relación entre dos contratos celebrados sucesivamente con un consumidor, que presentan entre ambos un fuerte vínculo -tal y como ha quedado de manifiesto al detallarse los hechos-, pero de los cuales sólo el primero se ha celebrado directamente en el ámbito de las actividades que dicho profesional dirige por medio de publicidad hacia el Estado miembro del domicilio del consumidor ${ }^{8}$.

\footnotetext{
${ }^{4}$ Vid. STJUE23 diciembre 2015, Rüdiger Hobohmc. Benedikt KampikLtd \& Co. KGyotros, C-297/14(ECLI:EU:C:2015:844), apartado 21.

5 Vid. Considerandos (11), (12) y (13) del Reglamento 44/2001.

${ }^{6}$ Se señala en principio porque como se verá en el fallo de esta sentencia, aunque el contrato de mandato no cumple de forma directa las condiciones del art. 15.1.c) del Reglamento 44/2001, sí se va a poder acudir al foro del domicilio del consumidor en virtud del art. 16.1 del Reglamento 44/2001, siempre que el contrato de mandato satisfaga "indirectamente" las condiciones del art. 15.1.c) del Reglamento 44/2001. A lo largo del presente artículo, se irá concretando lo que debe entenderse por "indirectamente", a la hora de determinar si el contrato de mandato cumple con los requisitos del art. 15.1.c) del Reglamento 44/2001.

7 STJUE 7 diciembre 2010, Peter Pammer c. Reederei Karl Schlüter GmbH \& Co. KG y Hotel Alpenhof GesmbH c. Oliver Heller, C-585/08 y C-144/09, Rec. 2010, p. I-12527 (ECLI:EU:C:2010:740); STJUE 6 septiembre 2012, Daniela Mühlleitner c. Ahmad Yusufi y Wadat Yusufi, C-190/11 (ECLI:EU:C:2012:542); STJUE 17 octubre 2013, Lokman Emrek c. Vlado Sabranovic, C-218/12 (ECLI:EU:C:2013:666); STJUE 14 noviembre 2013, Armin Maletic y Marianne Maletic c. lastminute.com GmbH y TUI Österreich GmbH, C-478/12 (ECLI:EU:C:2013:735).

8 Conclusiones del Abogado General, Pedro Cruz Villalón, presentadas el 8 de septiembre de 2015, Rüdiger Hobohm c. Benedikt Kampik Ltd \& Co. KG y otros, C-297/14 (ECLI:EU:C:2015:556), apartado 4.
} 


\section{Los requisitos para la aplicación del artículo 15.1.c) del Reglamento 44/2001 (art. 17.1.c) del Reglamento 1215/2012)}

10. Como ya se ha dicho el Tribunal de Justicia ha interpretado el art. 15.1 del Reglamento 44/2001 en varias ocasiones, señalando que éste es aplicable cuando se cumplen cumulativamente tres requisitos: en primer lugar, que una de las partes en el contrato tenga la condición de consumidor, de manera que actúe en un contexto totalmente diferente al de su actividad profesional; en segundo lugar, que se haya celebrado realmente el contrato entre dicho consumidor y el profesional: $y$, en tercer lugar, que el contrato pertenezca a una de las categorías incluidas en el apartado 1, letras a), b) y c), del artículo $15^{9}$.

11. De la resolución del Bundesgerichtshof se puede deducir que el primer requisito se cumple ya que el Sr. Hobohm y su esposa actuaron como consumidores finales privados, de hecho este aspecto no es objeto de discusión en el asunto que se plantea ${ }^{10}$.

También en la citada resolución se pone de manifiesto que la otra parte contratante por el contrario, actuaba en el ejercicio de sus actividades profesionales. Aunque el Reglamento 44/2001 no contiene esta exigencia, el TJUE estima que sólo hay contrato de consumo si el otro contratante es un profesional y no una persona que opera a título particular ${ }^{11}$. En opinión de la doctrina esta interpretación presenta la ventaja de que el Reglamento 44/2001 va en la misma línea que el Reglamento que regula la ley aplicable a las obligaciones contractuales, conocido como Reglamento Roma I, el cual en su art. 6.1, exige de modo expreso que para que sea considerado un contrato de consumo es obligatorio que la otra parte sea un profesional ${ }^{12}$.

12. En lo que respecta al segundo de los requisitos, tal y como se ha detallado en los hechos, hay constancia de que el matrimonio Hobohm y el Sr. Kampik celebraron el contrato de mandato en el año $2008^{13}$.

\footnotetext{
9 STJUE 14 marzo 2013, Česká spořitelna, a.s. c. Gerald Feichter, C-419/11 (ECLI:EU:C:2013:165), apartado 30; STJUE 28 enero 2015, Harald Kolassa contra Barclays Bank plc., C-375/13 (ECLI:EU:C:2015:37), apartado 23.

10 STJUE 14 marzo 2013, Česká spořitelna, a.s. c. Gerald Feichter, C-419/11 (ECLI:EU:C:2013:165), apartado 32; STJUE 5 diciembre 2013, Walter Vapenik c. Josef Thurner, C-508/12 (ECLI:EU:C:2013:790), apartado 28. Para un análisis de la noción de consumidor en el ámbito del Reglamento 44/2001, puesto que se trata de un concepto autónomo, Vid. H. GAUDEMET-TAllon, Compétence et exécution des jugements en Europe, Règlement 44/2001, Conventions de Bruxelles (1968) et de Lugano (1988 et 2007), $4^{a}$ Edición, LGDJ, París, 2010, pp. 287-291; E. Castellanos Ruiz, Régimen jurídico de los consumidores: competencia judicial internacional y ley aplicable, Comares, Granada, 2010, pp. 8-10; P. ARNT NielsEN, "Jurisdiction over consumer contracts", en U. MAGnUS/ P. MANKOWSKI (dirs.), Brussels I Regulation on Jurisdiction and Recognition in Civil and Commercial Matters, European Commentaries on Private International Law, $2^{a}$ Edición, Selp, Munich, 2012, pp. 374-376; J. Carrascosa González, "Operaciones internacionales de consumo”, en A.-L. Calvo Caravaca/ J. Carrascosa González (dirs.), Derecho Internacional Privado, Volumen II, Comares, Granada, 2016, p. 1105.

11 STJUE 28 enero 2015, Harald Kolassa contra Barclays Bank plc., C-375/13 (ECLI:EU:C:2015:37), apartados 22-25. En este sentido Vid. E. Castellanos Ruiz, Régimen jurídico de los consumidores: competencia judicial internacional y ley aplicable, Comares, Granada, 2010, pp. 10-11; P. ARnt Nielsen, "Jurisdiction over consumer contracts", en U. MAGNuS/ P. Mankowski (dirs.), Brussels I Regulation on Jurisdiction and Recognition in Civil and Commercial Matters, European Commentaries on Private International Law, $2^{\text {a }}$ Edición, Selp, Munich, 2012, pp. 376-377; J. Carrascosa GonzÁlez, "Operaciones internacionales de consumo”, en A.-L. Calvo Caravaca/ J. Carrascosa GonzÁlez (dirs.), Derecho Internacional Privado, Volumen II, Comares, Granada, 2016, p. 1106.

12 E. Castellanos Ruiz, Régimen jurídico de los consumidores: competencia judicial internacional y ley aplicable, Comares, Granada, 2010, pp. 10-11; J. CARrascosa GonzÁlez, "Operaciones internacionales de consumo", en A.-L. Calvo CaravaCa/ J. Carrascosa González (dirs.), Derecho Internacional Privado, Volumen II, Comares, Granada, 2016, p. 1106. Se debe añadir que el Reglamento 1215/2012 ha introducido una importante novedad con respecto al Reglamento 44/2001, y es que para la aplicación de los foros de protección de consumidores no es necesario que el profesional demandado esté domiciliado en un Estado miembro (J. Carrascosa González, "Operaciones internacionales de consumo", en A.-L. Calvo Caravaca/ J. Carrascosa GonzÁlez (dirs.), Derecho Internacional Privado, Volumen II, Comares, Granada, 2016, p. 1106). De manera que en el asunto que nos ocupa, como el profesional demandado, el Sr. Kampik, está domiciliado en España, es de aplicación el Reglamento 44/2001 pues se cumple el ámbito de aplicación personal -demandado domiciliado en un Estado miembro-, salvo las excepciones que recoge el Reglamento 44/2001, como son los foros exclusivos o la sumisión tácita, en los que no se exige dicho requisito para la aplicación del Reglamento 44/2001. Pues bien, a partir de la entrada en vigor del Reglamento 1215/2012, aunque el profesional demandado no esté domiciliado en un Estado miembro, sino en un tercer Estado, los foros de protección de consumidores que también contempla el Reglamento 1215/2012 sí que son de aplicación.

13 STJUE 14 marzo 2013, Česká spořitelna, a.s. c. Gerald Feichter, C-419/11 (ECLI:EU:C:2013:165), apartado 30; STJUE 28 enero 2015, Harald Kolassa contra Barclays Bank plc., C-375/13 (ECLI:EU:C:2015:37), apartados 29-34.
} 
13. En cuanto al tercer requisito, en virtud del artículo 15.1.c) del Reglamento $44 / 2001$ es necesario que, para que el contrato de mandato pueda quedar comprendido en el ámbito de aplicación de dicha disposición, deben cumplirse dos condiciones. Es necesario, por un lado, que el profesional realice actividades comerciales o profesionales en el Estado miembro del domicilio del consumidor (Doing Business Rule) o que, por cualquier medio, dirija tales actividades a dicho Estado miembro o a varios Estados miembros, incluido este último (Stream-of-Commerce Rule/ Country-of-Destination Rule); y, por otro lado, que el contrato de que se trate esté comprendido en el marco de dichas actividades ${ }^{14}$. Por lo tanto, los interrogantes planteados por el Bundesgerichtshof incumben a la parte de la disposición mencionada que se refiere al contrato celebrado en el marco de una actividad comercial o profesional "dirigida" por un profesional al Estado miembro del domicilio del consumidor.

14. En el presente asunto, si bien el contrato de intermediación se hallaba comprendido en el marco de la actividad de intermediación inmobiliaria "dirigida a" Alemania por el Sr. Kampik, no sucedía lo mismo con el contrato de mandato, si es considerado de forma separada e independiente del contrato de intermediación, tal y como señala acertadamente en sus Conclusiones el Abogado General, Pedro Cruz Villalón ${ }^{15}$.

Sin embargo, el Bundesgerichtshof estima que entre el contrato de intermediación y el contrato de mandato existe un nexo que deriva de la identidad de su finalidad económica y que justifica que este último contrato quede también comprendido en el ámbito de aplicación del artículo 15.1.c) del Reglamento 44/2001, al igual que el contrato de intermediación.

Por lo tanto, en la sentencia objeto de estudio se procede a examinar, en virtud del artículo 15.1.c) del Reglamento 44/2001 y de los objetivos que se propone este mismo Reglamento, si la existencia de un nexo entre los contratos mencionados puede llevar a que se considere que el contrato de mandato se enmarca en la actividad "dirigida a" Alemania por el Sr. Kampik y, en caso de que así sea, cuál debe ser la naturaleza de tal nexo.

Para dar respuestas a estas interrogantes se va a estudiar en primer lugar, lo que se entiende por "actividad dirigida" y en segundo lugar, cuáles deben ser las cualidades del nexo entre los dos contratos, que permitan afirmar que el contrato de mandato se enmarca dentro de la "actividad dirigida" arriba señalada, cumpliendo así con los requisitos para que sea de aplicación el art. 15.1.c) del Reglamento 44/2001.

\section{El concepto de "actividad dirigida"}

15. El concepto de "actividad dirigida" no se encuentra definido en el Reglamento 44/2001, de manera que surgen problemas a la hora de determinar cuáles son las "actividades dirigidas a un país concreto" 16 . Sin embargo, las "actividades dirigidas" se verifican, al menos en dos supuestos: a) el profesional dirige $e$-mails publicitarios a potenciales consumidores radicados en otro Estado; b) el profesional utiliza ciertos websites mediante los cuales intenta penetrar en un mercado nacional concreto, captar clientes, hacer negocios, por ejemplo porque realiza publicidad e incita a la contratación a los sujetos en un país concreto ${ }^{17}$. Ahora bien, no todas las websites van a implicar necesariamente "actividades dirigidas", sino que la website debe presentar carácter comercial, estar orientada hacia determinados Estados, así como los datos presentes en la website pueden permitir averiguar si el empresario desea conquistar el mercado de un país determinado, como que la website esté redactada en un determinado idioma que

\footnotetext{
14 STJUE 6 septiembre 2012, Daniela Mühlleitner c. Ahmad Yusufi y Wadat Yusufi, C-190/11 (ECLI:EU:C:2012:542), apartado 36.

${ }^{15}$ Conclusiones del Abogado General, Pedro Cruz Villalón, presentadas el 8 de septiembre de 2015, Rüdiger Hobohm c. Benedikt Kampik Ltd \& Co. KG y otros, C-297/14 (ECLI:EU:C:2015:556), apartado 33.

16 E. Castellanos Ruiz, Régimen jurídico de los consumidores: competencia judicial internacional y ley aplicable, Comares, Granada, 2010, p. 128.

17 E. Castellanos Ruiz, Régimen jurídico de los consumidores: competencia judicial internacional y ley aplicable, Comares, Granada, 2010, p. 127; J. CARrascosa GonzÁlez, "Operaciones internacionales de consumo”, en A.-L. Calvo Caravaca/ J. Carrascosa González (dirs.), Derecho Internacional Privado, Volumen II, Comares, Granada, 2016, p. 1115.
} 
sólo se habla en algunas zonas del planeta, que aparezca la moneda de pago exigida, que los teléfonos se reflejen con prefijos internacionales, etc ${ }^{18}$.

En este sentido, se sigue la tesis de la "focalización" en la que sólo es relevante la voluntad del profesional de dirigir sus actividades hacia el público de un determinado país y que dicha voluntad sea perceptible por el consumidor radicado en un concreto país ${ }^{19}$.

Si se analizan los hechos y teniendo en cuenta el concepto de "actividad dirigida" señalado se puede afirmar que ha existido una "actividad dirigida" hacia Alemania por parte del Sr. Kampik, en relación con el contrato de intermediación, pero no se puede afirmar lo mismo en lo referente al contrato de mandato. En cualquier caso, debe ser el órgano jurisdiccional nacional quien debe efectuar una apreciación global de las circunstancias en las que fue celebrado el contrato de consumo controvertido en el litigio principal para decidir si, en función de la existencia o no de indicios que figuren o no en la lista no exhaustiva según la jurisprudencia, es aplicable el artículo 15.1.c), del Reglamento 44/200120.

16. Ahora bien en el caso que nos ocupa, ¿cuál debe ser la línea interpretativa a seguir de dicho concepto en relación con el contrato de mandato?, es decir, ¿se debe realizar una interpretación amplia o bien, una interpretación estricta del mismo, para poder determinar si el contrato de mandato señalado cumple los requisitos del art. 15.1.c) del Reglamento 44/2001, aunque no se halle comprendido en el ámbito de la "actividad dirigida" por ese profesional al Estado miembro del domicilio del consumidor, pero que presenta un nexo estrecho con el contrato de intermediación celebrado en el marco de dicha actividad?. Tal y como se recoge en la sentencia, el TJUE en distinta jurisprudencia ha señalado la necesidad de interpretar de forma autónoma los conceptos utilizados por el Reglamento 44/2001, con referencia a su sistema y objetivos ${ }^{21}$. Como se desprende de los considerandos (11), (13) y (15) del Reglamento 44/2001, forman parte de estos objetivos, la previsibilidad de las reglas de competencia, la protección del consumidor y la reducción de la posibilidad de procedimientos paralelos, con el fin de evitar que se dicten en dos Estados miembros resoluciones inconciliables ${ }^{22}$.

En cuanto a la protección del consumidor, de la jurisprudencia del Tribunal de Justicia relativa al régimen particular establecido por las disposiciones del Convenio de Bruselas, también aplicable con respecto a las disposiciones equivalentes del Reglamento 44/2001, se desprende que los preceptos señalados tienen como fin garantizar la adecuada protección del consumidor, en comparación con el profesional con quien contrata ${ }^{23}$. Si se tiene en cuenta dicho objetivo, debe realizarse una interpretación amplia del art. 15.1.c) del Reglamento 44/2001.

18 Para un análisis de los datos presentes en la website para saber si un profesional desea conquistar un determinado mercado Vid. STJUE 7 diciembre 2010, Peter Pammer c. Reederei Karl Schlüter GmbH \& Co. KG y Hotel Alpenhof GesmbH c. Oliver Heller, C-585/08 y C-144/09, Rec. 2010, p. I-12527 (ECLI:EU:C:2010:740), apartados 73-80 y 93.

19 Tal y como explican U. Magnus/ P. MANKowski, a través de una metáfora, el empresario que opera en Internet es un pescador (angler) que trata de pescar al consumidor (the fish) utilizando una caña de pescar (fishing rid). Es decir que lo importante es que el profesional toma la iniciativa comercial y comprobar si el consumidor le responde (H. GAUdEMET-TALLON, Compétence et exécution des jugements en Europe, Règlement 44/2001, Conventions de Bruxelles (1968) et de Lugano (1988 et 2007), $4^{\mathrm{a}}$ Edición, LGDJ, París, 2010, pp. 294-296; E. Castellanos RuIz, Régimen jurídico de los consumidores: competencia judicial internacional y ley aplicable, Comares, Granada, 2010, pp. 128-131; P. ARNT NIELSEN, "Jurisdiction over consumer contracts", en U. Magnus/ P. Mankowski (dirs.), Brussels I Regulation on Jurisdiction and Recognition in Civil and Commercial Matters,

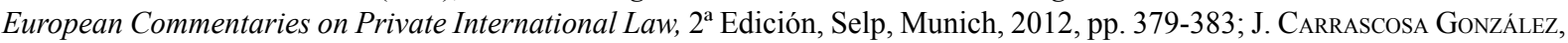
"Operaciones internacionales de consumo", en A.-L. Calvo Caravaca/ J. CARrascosa González (dirs.), Derecho Internacional Privado, Volumen II, Comares, Granada, 2016, pp. 1114-1116).

20 Vid. STJUE 17 octubre 2013, Lokman Emrek c. Vlado Sabranovic, C-218/12 (ECLI:EU:C:2013:666), apartado 31.

${ }^{21}$ STJUE 7 diciembre 2010, Peter Pammer c. Reederei Karl Schlüter GmbH \& Co. KG y Hotel Alpenhof GesmbH c. Oliver Heller, C-585/08 y C-144/09, Rec. 2010, p. I-12527 (ECLI:EU:C:2010:740), apartado 55; STJUE 14 marzo 2013, Česká sporitelna, a.s. c. Gerald Feichter, C-419/11 (ECLI:EU:C:2013:165), apartado 25.

22 Sobre los objetivos de protección del consumidor y de reducción de la posibilidad de procedimientos paralelos Vid. STJUE 6 septiembre 2012, Daniela Mühlleitner c. Ahmad Yusufi y Wadat Yusufi, C-190/11 (ECLI:EU:C:2012:542), apartado 29; STJUE 14 noviembre 2013, Armin Maletic y Marianne Maletic c. lastminute.com GmbH y TUI Österreich GmbH, C-478/12 (ECLI:EU:C:2013:735), apartado 30.

23 STJUE 14 marzo 2013, Česká spořitelna, a.s. c. Gerald Feichter, C-419/11 (ECLI:EU:C:2013:165), apartado 33; STJUE 28 enero 2015, Harald Kolassa contra Barclays Bank plc., C-375/13 (ECLI:EU:C:2015:37), apartado 21. 
No obstante, en la sentencia también se señala que el artículo 15.1.c) del Reglamento 44/2001 constituye una excepción tanto a la regla general de competencia señalada en el artículo 2.1 de dicho Reglamento, que atribuye competencia a los órganos jurisdiccionales del Estado miembro del domicilio del demandado, como a la regla de competencia especial en materia de contratos, contenida en el artículo 5.1 de dicho Reglamento, según la cual el tribunal competente es el del lugar en el que haya sido o deba ser cumplida la obligación que sirva de base a la demanda ${ }^{24}$. En este sentido, dicha disposición ha de ser obligatoriamente objeto de una interpretación estricta. Esta misma línea interpretativa resulta de la jurisprudencia del Tribunal de Justicia, en la que se afirma que si bien el artículo 15.1.c) del Reglamento 44/2001 tiene por objeto proteger a los consumidores, esto no implica que esa protección sea absoluta ${ }^{25}$.

17. Tras dicho análisis, en la sentencia que nos ocupa, se llega a la conclusión de que el artículo 15.1.c) de dicho Reglamento puede aplicarse a un contrato, como el contrato de mandato objeto del litigio principal, que presente un nexo estrecho con otro contrato, de las características del contrato de intermediación. Ahora bien, ¿cuáles son los elementos constitutivos del "nexo estrecho" entre los dos contratos?

\section{Los elementos constitutivos de "nexo estrecho"}

18. En cuanto a la posible presencia de elementos constitutivos de nexo estrecho antes señalado, consta en este caso, que a raíz de la insolvencia del promotor quedó frustrado "el objetivo económico que subyacía al contrato de intermediación", que era poder comprar una casa en España, gracias a la actividad de intermediación inmobiliaria "dirigida" por el Sr. Kampik "al" Estado miembro del domicilio del matrimonio Hobohm. Precisamente para subsanar el incumplimiento de ese objetivo económico y procurar al matrimonio Hobohm, en su condición de consumidores, la prestación que era objeto de dicha actividad, el Sr. Kampik, les propuso la celebración de un contrato de mandato. La finalidad de este contrato era, pues, alcanzar "el objetivo económico concreto que subyacía al contrato de intermediación"26.

Por lo anterior se señala en la sentencia que el contrato de mandato, aun cuando no se inscriba como tal en el ámbito de la actividad comercial o profesional dirigida, se celebró "como prolongación directa de dicha actividad y es complementario del contrato de intermediación, dado que su finalidad es la satisfacción del objetivo económico que subyace a este último".

En consecuencia, aunque no existe interdependencia jurídica entre el contrato de intermediación y el contrato de mandato, el nexo económico existe. Este nexo reside en la "satisfacción del objetivo económico que subyace al contrato de intermediación", que no era otro que garantizar el disfrute efectivo del apartamento cuya terminación peligraba por la insolvencia del promotor. Al no realizarse las obras de terminación pactadas entre las partes en virtud del contrato de mandato, el disfrute efectivo del apartamento no fue posible.

19. Además, en el fallo de la sentencia se añade que si existe un nexo estrecho entre el contrato de mandato y el contrato de intermediación, el órgano jurisdiccional nacional debe tener en cuenta los elementos constitutivos de ese nexo, concretamente: "la identidad fáctica o jurídica de las partes de los dos contratos, la identidad del objetivo económico que ambos contratos, orientados al mismo objeto concreto, se proponen conseguir y la complementariedad del contrato de mandato con respecto al contrato de intermediación, en la medida en que tiene por fin facilitar la satisfacción del objetivo económico subyacente a este último contrato". Por lo que, será el juez nacional quien deba valorar los elementos constitutivos del nexo estrecho entre ambos contratos.

20. Por último, en la sentencia se destaca que, en relación con la garantía de previsibilidad de las normas de competencia judicial del considerando (11) del Reglamento 44/2001, en el caso planteado, el

${ }^{24}$ STJUE 28 enero 2015, Harald Kolassa contra Barclays Bank plc., C-375/13 (ECLI:EU:C:2015:37), apartado 28.

25 STJUE 6 septiembre 2012, Daniela Mühlleitner c. Ahmad Yusufi y Wadat Yusufi, C-190/11 (ECLI:EU:C:2012:542), apartado 33 .

26 Siguiendo esta misma línea interpretativa Vid. J. CARrascosa GonzÁlez, "Operaciones internacionales de consumo", en A.-L. Calvo Caravaca/ J. Carrascosa González (dirs.), Derecho Internacional Privado, Volumen II, Comares, Granada, 2016, p. 1113. 
foro del domicilio del consumidor sería competente para conocer de un litigio relativo a un contrato de intermediación que se enmarca en la actividad del profesional "dirigida al" Estado miembro del domicilio del consumidor. Si posteriormente este profesional celebra con el mismo consumidor un contrato para alcanzar el objetivo fundamental que se pretendía conseguir con el primer contrato, parece razonable que cuente con que ambos contratos queden sujetos al mismo sistema de competencia judicial.

\section{Conclusiones}

21. Una vez expuesto lo anterior, el fallo de la sentencia es a favor de la aplicación del artículo 15.1.c) del Reglamento 44/2001, en relación con el artículo 16.1 de dicho Reglamento, cuando se trate de un contrato, celebrado entre un consumidor y un profesional, que no se halla comprendido como tal en el ámbito de la actividad comercial o profesional "dirigida" por ese profesional «al» Estado miembro del domicilio del consumidor, pero que presenta un nexo estrecho con un contrato celebrado anteriormente entre las mismas partes en el marco de dicha actividad. Y será el órgano jurisdiccional nacional quien verifique si concurren los elementos constitutivos de ese nexo, en particular los que se han relacionado en el parágrafo $19^{27}$.

22. En mi opinión la sentencia es acertada tanto en el fallo como en los motivos en los que se fundamenta dicha sentencia. El nexo entre ambos contratos es determinante en el fallo a favor de la aplicación del foro de consumidores a ambos contratos ${ }^{28}$. Si bien los problemas que puedan surgir serán en relación con los elementos constitutivos del nexo.

Efectivamente en el supuesto planteado, está bastante claro el nexo entre los dos contratos que deriva de que ambos tienen la misma finalidad económica, la adquisición de la vivienda o el uso y disfrute de la misma.

Sin embargo, en los litigios que surgan con posterioridad a esta sentencia, es posible que el nexo entre los contratos en cuestión no sea tan claro, ni manifiesto; y que el supuesto planteado presente ciertas particularidades, de forma que los elementos constitutivos del nexo entre ambos contratos sean distintos a los señalados en esta sentencia. En cuyo caso, habrá que realizar un análisis similar al presente, estudiando en primer lugar, si el profesional ha realizado "actividades dirigidas" hacia el consumidor y, en segundo lugar, cuáles son los elementos constitutivos del nexo estrecho entre los dos contratos. Y poder determinar si efectivamente son de aplicación los arts. 15.1.c) y 16.1 del Reglamento 44/2001.

Por último señalar, que en el párrafo final del fallo se elige a los tribunales nacionales como órganos encargados de comprobar que efectivamente se cumplen los elementos constitutivos de dicho nexo estrecho y que son exactamente: "la identidad fáctica o jurídica de las partes de los dos contratos, la identidad del objetivo económico que ambos contratos, orientados al mismo objeto concreto, se proponen conseguir y la complementariedad del segundo contrato con respecto al primero, en la medida en que tiene por fin facilitar la satisfacción del objetivo económico subyacente a este último contrato"29. Me parece adecuado que sean precisamente los órganos jurisdiccionales nacionales los que pongan los medios para corroborar que se cumplen los elementos constitutivos del nexo estrecho, los cuales podrán comprobar in situ si se verifican o no.

${ }^{27}$ Si bien el Abogado General, Pedro Cruz Villalón, llega al mismo resultado en sus Conclusiones que el fallo de la sentencia de autos, la fundamentación es diferente. Vid. Conclusiones del Abogado General, Pedro Cruz Villalón, presentadas el 8 de septiembre de 2015, Rüdiger Hobohm c. Benedikt Kampik Ltd \& Co. KG y otros, C-297/14 (ECLI:EU:C:2015:556), apartado 56.

${ }_{28}$ Aunque no puedo estar de acuerdo con los argumentos señalados en las Conclusiones del Abogado General, Pedro Cruz Villalón, que aunque aborda el tema de la relación causal entre ambos contratos, dicha vinculación es analizada como un indicio de la "actvidad dirigida" (Conclusiones del Abogado General, Pedro Cruz Villalón, presentadas el 8 de septiembre de 2015, Rüdiger Hobohm c. Benedikt Kampik Ltd \& Co. KG y otros, C-297/14 (ECLI:EU:C:2015:556), apartados 39-45).

29 Tampoco coincide este apartado final del fallo de la sentencia con el párrafo final de las Conclusiones del Abogado General, Pedro Cruz Villalón, donde se señala de manera literal que: "en el caso de que la jurisdicción nacional determine que ha existido una oferta por parte del profesional al consumidor, ha de considerarse que dicha oferta entra dentro de "cualquier medio " a través del cual un profesional puede dirigir su actividad al Estado miembro del domicilio del consumidor". Pero todo ello es debido a que el Sr. Kampik ofreció al demandante encargarse de la habitabilidad de la vivienda, lo cual no está en absoluto relacionado con el nexo estrecho de los contratos, que es el argumento fundamental para la aplicación del art. 15.1.c) y 16.1 del Reglamento 44/2001 (Conclusiones del Abogado General, Pedro Cruz Villalón, presentadas el 8 de septiembre de 2015, Rüdiger Hobohm c. Benedikt Kampik Ltd \& Co. KG y otros, C-297/14 (ECLI:EU:C:2015:556), apartados 47-56). 